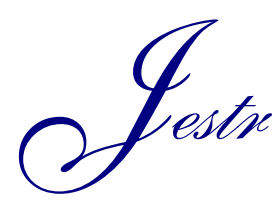

Journal of Engineering Science and Technology Review 9(6) (2016) 116- 121

Research Article

\title{
Method of Analysis and correction of the Error from Nonlinearity of the Measurement Instruments
}

\author{
D. Dichev ${ }^{1, *}$, F. Kogia ${ }^{1}$, Hr. Koev ${ }^{1}$ and D. Diakov ${ }^{2}$ \\ ${ }^{1}$ Technical University-Gabrovo, 5300 Gabrovo, 4 H. Dimitar Str., Bulgaria \\ ${ }^{2}$ Technical University-Sofia, 1000 Sofia, 8 Kl. Ohridski Blvd., Bulgaria
}

Received 24 November 2016; Accepted 29 December 2016

\begin{abstract}
In this work are examined the characteristics of the error from nonlinearity of the static characteristic of the measuring instruments. The possibilities to correct this error are analyzed. Mathematical models are presented, in order to allow evaluation of the error from nonlinearity regarding the permissible error of the measurement result, as well as to draw the necessary algorithms for correction of this error. The models are developed, based on the method of the smallest modules.
\end{abstract}

Keywords: measuring device; error from nonlinearity; static characteristic of a measuring instrument; error evaluation; error correction

\section{Introduction}

The static characteristic of the measuring instruments is determined by the relationship between the informative parameters of signals at the input and output of the devices, operating in static mode of measurement [1]. Usually, the measurement instruments have a linear static characteristic. The linear mathematical model of static characteristic of the measuring instruments is defined by two parameters and is suitable for practical use because of several advantages, the most important of which are:

1. The coefficients $a$ and $k$ have a clear physical significance and can easily associate in a theoretical aspect with the structural parameters of the measuring instruments. Whereupon, the $a$ coefficient, characterizing the shift of the static characteristic from zero, and $k$ is the coefficient of sensitivity of the measuring device.

2. In a linear static characteristic procedure, an "exclusion" of the systematic error is significantly simplified if the estimates and coefficients $\tilde{a}$ and $\tilde{k}$. In this case, the structure of the possible outcome of the measurement can be written as

$y(x)=\widetilde{a}+\widetilde{k} \cdot x+\stackrel{o}{\varepsilon}$,

$o$

where $\varepsilon$ is the possible value of the centered random error o

After solving the equation (1), for $x$ is obtained:

$x=\frac{1}{\widetilde{k}} \cdot[y(x)-\tilde{a}-\varepsilon] \approx \frac{1}{\widetilde{k}} \cdot[y(x)-\tilde{a}]=\tilde{x}=\hat{y}(x)$,

- E-mail address: dichevd@abv.bg

ISSN: 1791-2377 @ 2016 Eastern Macedonia and Thrace Institute of Technology. All rights reserved. where $\tilde{x}=\hat{y}(x)$ is the assessment of the measured value $x$, determined by the corrected measurement result $\hat{y}(x)$.

3. Using linear static characteristic, an opportunity is provided for easy conversion of the characteristics of the systematic error in the specific requirements towards the design parameters of the measuring equipment.

4. The linear model of static characteristic has a very important property superposition. This property can be described mathematically as follows. Let $a=0$, and the measured value is equal to $x=x_{I}+x_{2}$. Yielding:

$y(x)=k \cdot x=k \cdot\left(x_{1}+x_{2}\right)=k \cdot x_{1}+k \cdot x_{2}=y\left(x_{1}\right)+y\left(x_{2}\right)$.

From (3), and the property of superposition, it follows that the result of the measuring of the sum of the values $x_{I}$ and $x_{2}$, with an accuracy to the shift the zero $a$, is equal to the sum of the measurement results of each of the two values individually. Superposition provides an opportunity for a summation of errors, caused by various confounding factors, it simplifies the procedures for conducting such important for the metrology events, like the verification of the measuring instruments and others.

Due to the above mentioned advantages, the majority of the measuring instruments have a linear nominal static characteristic.

On the other hand, due to a number of instrumental and systematic errors, existing in the structures of the measuring instruments, and as a result of some confounding external and internal factors, the actual static characteristic differs from the nominal [1]. Usually, the actual static characteristic has a non-linear form. Then, the difference between the actual and nominal static characteristics determines the error from nonlinearity of the measuring instrument.

For the most part, modern measuring devices have the necessary hardware part for the introduction of algorithms for optimal construction of the linear static, in regards to the actual, so that the error from nonlinearity has minimum values $[2,3]$. This is an important and promising task in the 
metrology science, whose effective resolution leads to an increases of the accuracy of measurement.

2. Analysis of the structural scheme of measuring instruments in regards to the opportunities for correction of the error from nonlinearity
A distinctive feature of the modern stage of development of the measurement technology is the expansion of the capabilities of the measuring instruments in relation to the response time and the volume of processed information, which is based primarily on the rapid development of the microprocessor and computer equipment $[4,5]$. In light of the foregoing, the majority of current measuring devices are built on the basis of the structural diagram, shown in fig. 1 .

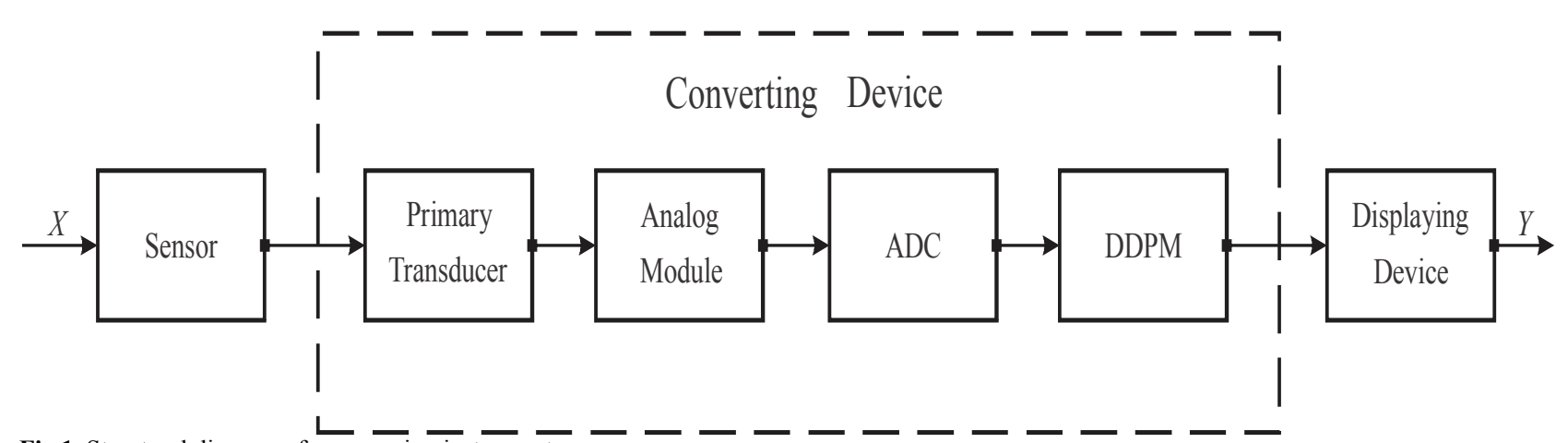

Fig.1. Structural diagram of a measuring instrument

The structural scheme consists of a sensor, subjected to the direct impact of the measured value, primary converter, analog module, commonly used for the amplification and filtering of the signal, analog-to-digital converter (ADC), module for digital data processing (DDPM) and detection device.

The module for digital processing enables the implementation of a number of algorithms, related to improving the quality of the measurement information $[6,7$, $8,9]$. These algorithms can be based on mathematical models, allowing both the expansion of the types of the measurement information, and the increase of the accuracy of measurement [10]. It is through this module that algorithms for correction of the error from nonlinearity can be implemented.

Therefore, in modern measuring devices, the error from nonlinearity of the static characteristic is usually removed in the digital part of the device, where the corresponding algorithm for calculation of the measurement result is carried out [11]. Therefore, as a basic condition, related to the metrological characteristics of measuring instruments, is considered less the linearity requirements of the static characteristic in the hardware part of the device, as its stability, ie, the immutability of this feature in the changing conditions of actual use [12,13].

\section{Mathematical model for the identification and correction of the errors from nonlinearity}

For the realization of the necessary algorithms for the identification and correction of the errors from nonlinearity of the static characteristics, corresponding mathematical models that adequately reflect the characteristics of this error should be drawn up. The error from nonlinearity has different values in the range of measurement, which are defined by the coordinate of the measured value, using the function of the error $\varepsilon=\varphi(x)$. From a methodological point of view, the problems, associated with the nonlinearity of the static characteristic, pose to solve two basic metrology tasks [14]:
- task to determine the errors from nonlinearity;

- task to correct the errors from nonlinearity.

The task of the determination of the errors from nonlinearity is solved by finding the function $\varepsilon=\varphi(x)$ that is based on the absolute differences between the graphically expressed functions of the actual characteristic, $y=f_{r}(x)$ and the approximating straight line $y_{n}=A+B \cdot x$. The actual static characteristic $y=f_{r}(x)$ usually occurs experimentally, and the nominal (the specified, the desired) characteristic

$y=f_{n}(x)=A+B \cdot x$

is the straight line, drawn in a certain way, connected with the accepted criteria to ensure the accuracy of the measuring instrument.

The parameters $\mathrm{A}$ and $\mathrm{B}$ define the position of the straight line in the Cartesian coordinate system and, in practice, are identified by the coefficient $a$, characterizing the shift of the static characteristic from zero and the coefficient of sensitivity of the measuring device $k$. Depending on how the construction of the straight line $y_{n}=A+B \cdot x$, the error from nonlinearity can be estimated by the method of the maximal reduced error and by the method of the average quadratic reduced error. In the first case, the approximated straight is constructed by the method of the smallest modules, and the second - by the method of the smallest squares. The object of this work will be the first of these two methods.

Depending on the mode, with which the second problem is solved, it can be divided into two types

- task, solved in the design stage of the measuring device;

- task, solved by the algorithm for correction in the digital part of the measuring device.

The first of these two tasks is decided by calculations, which gives the nominal values of the coefficients $q_{i}=q_{i}^{n}$, corresponding to the nominal static function of the 
measuring device

$$
y=f_{n}\left(x, q_{1}^{n}, q_{2}^{n}, q_{3}^{n}, \ldots, q_{m}^{n}\right)
$$

After obtaining the necessary values of the coefficients $q_{i}=q_{i}^{n}$, the corresponding meanings of these functional parameters of the structure of the measuring device, upon which they depend, can be selected. Therefore, in this case, the task is solved in terms of the synthesis of the measuring device, in which nominal values of the coefficients $q_{1}^{n}, q_{2}^{n}, q_{3}^{n}, \ldots, q_{m}^{n}$ can be defined, in which static characteristic coincide completely with nominal or differ from it by an amount, not exceeding the permissible error from nonlinearity.

The second task can be solved with the aid of the digital processing module (Figure 1), allowing the introduction of the algorithms, with which the nominal function $y_{n}=A+B \cdot x$ may be constructed in advance or in real time, depending on the actual static characteristic, so as to correspond to a certain optimization criterion.

The linear function, constructed by the method of the smallest modules, is called the straight $y_{n}=A+B \cdot x$, whose maximum deviation from the curve of the actual static characteristic $y=f_{r}(x)$ in the range $x_{\min } \leq x \leq x_{\max }$ has a minimum value. The parameters of the straight line are defined namely from the condition of the minimal value of the maximum deviation from the curve $y=f_{r}(x)$, ie

$$
\varepsilon_{\text {mod }}^{\max }(A, B)=\max _{x_{\min } \leq x \leq x_{\max }}\left|f_{r}(x)-(A+B \cdot x)\right|=\min
$$

From (6) it is clear that the minimum of the implicitly set two variables function is achieved through an optimal choice of the arguments $A$ and $B$ that define the position of the straight in the Cartesian coordinate system and can be considered as the only functional parameters of the straight of the smallest modules $y_{n}=A+B \cdot x$.

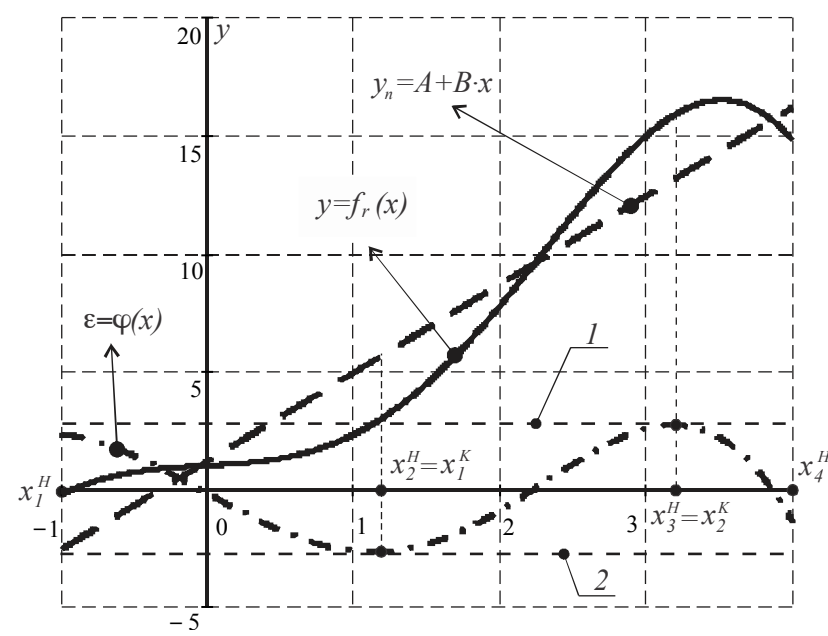

Fig.2. The straight line of the smallest modules and the function of the error

It can be proven that the maximum deviation has a minimum, when the straight $y_{n}(A, B)=A+B \cdot x$ is equidistant from the extremes of the error from nonlinearity.
The geometric interpretation of the concept equidistant is expressed in practice through such construction of a straight line of the static characteristic, whereby the maximum module values of the absolute error from nonlinearity are equal. This condition can be represented via the following system of $K+H-1$ nonlinear algebraic equations [1]:

$$
\begin{aligned}
& \delta_{1}=\delta_{2}=\delta_{3}=\ldots=\delta_{H}, \\
& \frac{d}{d x_{i}} \varepsilon\left(x_{i}, A, B\right)=0, \quad i=1,2,3, \ldots, K,
\end{aligned}
$$

where $\delta_{1}=\delta_{2}=\delta_{3}=\ldots=\delta_{H}$ - maximum module values of the absolute error from nonlinearity, whose number $H$ depends on the specifics of the function $y=f_{r}(x)$;

$$
\varepsilon(x, A, B)=f_{r}(x)-(A+B \cdot x) .
$$

- function, expressing successive values on the coordinate of the measured parameter $x$ on the absolute error from nonlinearity; $x_{i}$ - abscissae of these points from the actual static characteristic $y=f_{r}(x)$, where it has extrema; $K$ number of extrema, which depend on the characteristics of the function $y=f_{r}(x)$.

The second equation in the system (7) is based on the property of the derivative of a function at a point. As shown in fig.2, the tangent of the angles, whose tangents 1 and 2 to the function of the error $\varepsilon=\varphi(x)$ in the respective points conclude with the positive direction of the $\mathrm{x}$-axis, have zero values. The straight, defining the nominal static characteristic $y_{n}(A, B)=A+B \cdot x$, in fig.2 is constructed, according to the expressed through the system (7) condition of equidistance according to the actual static characteristic $y$ $=f_{r}(x)$. In this case, $K=2$ (the points with abscissae $x_{i}^{K}$ ), and $H=4$ (the points with abscissae $x_{j}^{H}$ ) (fig.2).

\section{Methods for calculating the parameters of the straight lines of the smallest modules}

In practice, the parameters $A$ and $B$, defining the straight line of the smallest modules can be calculated in two ways. The first is performed by solving the system of equations (7), expressing the condition of equidistance of the straight $y_{n}=A+B \cdot x$ in relation to the extrema of the function $y=$ $f_{r}(x)$. The second way consists in determining, on the basis of (6), these values of the parameters $A$ and $B$, in which the maximum difference between the nominal static characteristic $y_{n}=A+B \cdot x$ and the actual characteristic $y$ $=f_{r}(x)$ will have a minimum value.

The degree of complexity of the solution of the problem in the first way is determined primarily by the type of the actual static characteristic $y=f_{r}(x)$. Very often, in solving the problem in practice, the actual characteristic is broken down into a series connected simple functions, whose solutions can be defined easily by the above presented models. For example, if the actual characteristic is described by the function $f_{r}(x)=3,15 \cdot x^{2}$ for the interval $x_{\min }=0 \leq x$ $\leq 1=x_{\max }$, then it has a relatively simple solution in relation to the model (7). 


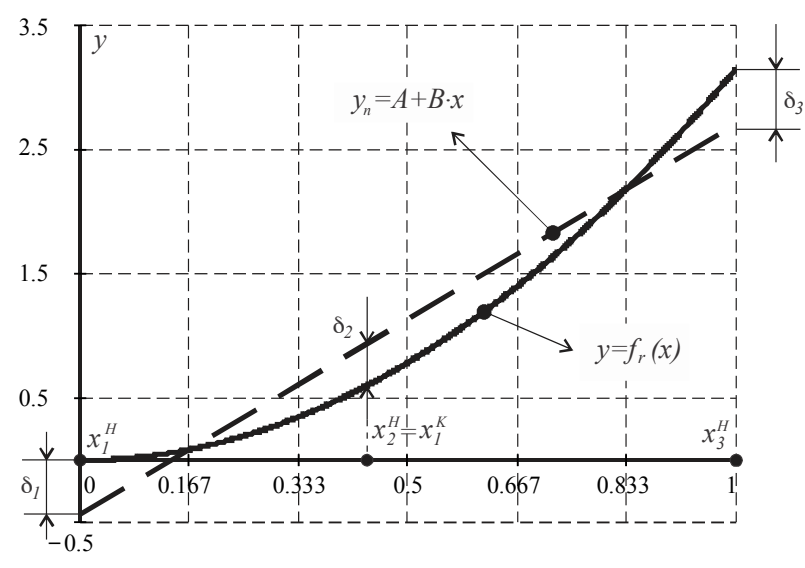

Fig. 3. The first way to construct the straight line of the smallest modules

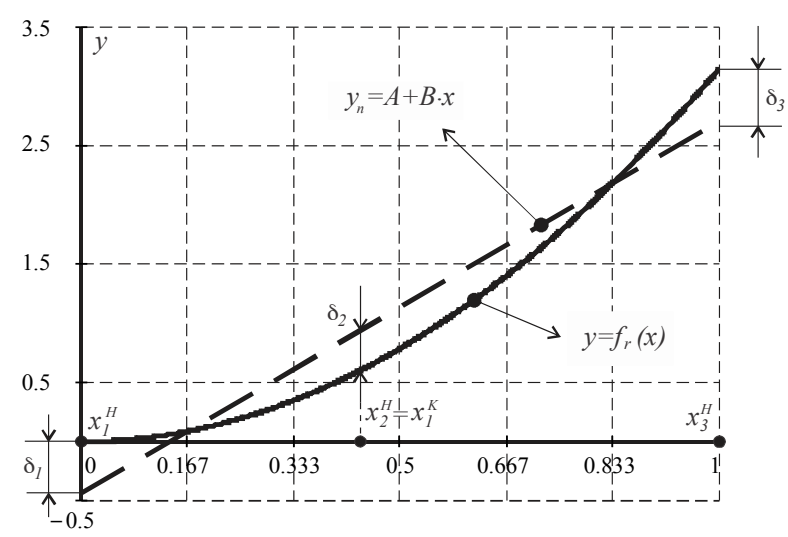

Fig. 4. A second way to build on the straight line of the smallest modules

The straight line of the smallest modules in this case can be built in two ways. In the first method, illustrated graphically in fig.3, achieves better results the condition of equidistance of the straight in relation to the function $y=$ $f_{r}(x)$ for the limits of the whole interval. In this case, the straight of the smallest modules will de $y_{n}=A+B \cdot x$, and the system (7) takes the following form:

$\delta_{1}=\delta_{2}$

$\delta_{1}=\delta_{3}$;

$\frac{d}{d x_{1}} \varepsilon\left(x_{1}, A, B\right)=\frac{d}{d x_{1}}\left(3,15 \cdot x_{1}^{2}-A-B \cdot x_{1}\right)$.

In the second method of construction, the graph of the straight passes through the origin of the coordinate system, where it intersects with the curve of the actual static characteristic $y=f_{r}(x)$, that at $x=0$ has a value of $f_{r}(0)=0$ (fig.4). This method has the important advantage of setting persistent initial conditions of the problem, which makes it easier to automate the process of constructing the straight of the smallest modules. Therefore, this task will be viewed in more detail.

Equation of the approximating straight will be

$y_{n}(x, B)=f_{n}(x)=B \cdot x ; A=0$,

since $f_{r}(0)=f_{n}(0)=0$ and $x_{\min }=0$.
The analytical expression of the function, defining the error from nonlinearity in this case will be

$\varepsilon(x, B)=3,15 \cdot x^{2}-B \cdot x$.

With the help of fig.4, the following condition (7) can be presented for the equidistance of the straight from the extrema of the error from nonlinearity as follows:

$$
\begin{aligned}
& \delta_{1}=\delta_{2} ; \\
& \frac{d}{d x_{1}} \varepsilon\left(x_{1}, B\right)=0 .
\end{aligned}
$$

where $\delta_{1}$ and $\delta_{2}$ are the maximum values of the module of the absolute error from nonlinearity; $x_{1}$ - the axis of the point, defining the extremum of this error.

In this case, based on fig.4, following expressions of the elements from (12) can be represented:

$\delta_{1}=-\varepsilon\left(x_{1}, B\right)=-3,15 \cdot x_{1}^{2}+B \cdot x_{1} ;$
$\delta_{2}=\varepsilon\left(x_{\max }, B\right)=3,15 \cdot x_{\max }^{2}-B \cdot x_{\max }=3,15-B ;$
$\frac{d}{d x_{1}} \varepsilon\left(x_{1}, B\right)=6,3 \cdot x_{1}-B$.

Then, based on (12), can be written

$-3,15 \cdot x_{1}^{2}+B \cdot x_{1}=3,15-B$

$6,3 \cdot x_{1}-B=0$.

Wherein $x_{1}=\frac{B}{6,3}$, after substitution in the first equation of (14), the following remains

$\frac{1}{2} \cdot \frac{B^{2}}{6,3}+B-3,15=0$.

From fig. 4, it follows that the negative root of the quadratic equation (15) makes no sense, therefore the searched for values will be $B=2,609$; $x_{1}=\frac{B}{6,3}=\frac{2,609}{6,3}=0,414$, and the equation of the straight of the smallest modules has the form

$y_{n}=2,609 \cdot x$

The graphical appearance of the location of those straight lines, in relation to the actual function of the static characteristic $f_{r}(x)=3,15 \cdot x^{2}$, is shown in fig.5.

Interesting from a methodological point of view is the case, when the actual function of the static characteristics can be divided into a series of simple curves, like in the discussed above example. In these cases, model (7) is difficult to apply because the decisions are considerably more complicated. For example, if the actual static characteristic of the measuring device is set by the function

$f_{r}(x)=x \cdot\left[e^{x \cdot \sin (2 \cdot x)}+2\right]$ in the interval 
$x_{\min }=-1,4 \leq x \leq 2=x_{\max }$,

then the derivative of this function significantly complicates the procedure for determining the parameters $A$ and $B$, according to model (7).

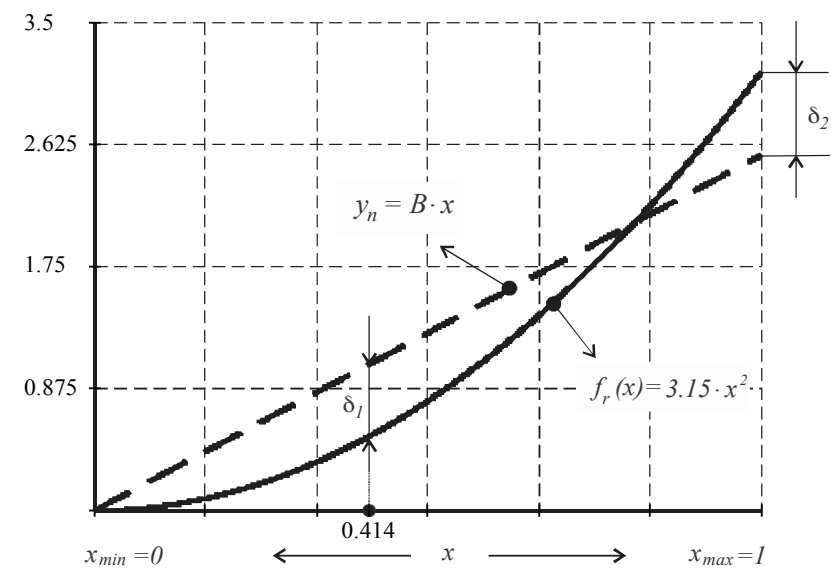

Fig. 5. Construction of the straight for the static characteristic

$f_{r}(x)=3,15 \cdot x^{2}$

Therefore it is necessary to solve the task in another way, based on other conditions, which on one hand have to simplify the calculation procedure, and on the other - not to change the concept of constructing a nominal static characteristic as the straight line of the smallest modules. It can be taken into consideration, in this context, the method, in which the approximated straight is constructed so that the average error from nonlinearity is zero. Such straight must meet the following condition:

$$
\bar{\varepsilon}=\int_{x_{\min }}^{x_{\max }} \varepsilon(x, A, B) \cdot d x=\int_{x_{\min }}^{x_{\max }}\left[f_{r}(x)-(A+B \cdot x)\right] \cdot d x=0 .
$$

From (18), a ratio to determine the parameter $A$ can easily be found

$$
A=\frac{1}{x_{\max }-x_{\min }} \cdot \int_{x_{\min }}^{x_{\max }} f_{r}(x) \cdot d x-\frac{B}{2} \cdot\left(x_{\max }+x_{\min }\right) .
$$

The determination of the parameter $B$ can be realized with different models, the most effective of which are made according to the shape of the curve, defining the actual static characteristic $y=f_{r}(x)$. One of these models consists in the determination of such two points, situated within the measuring range, in which the error from nonlinearity (8) is equal to zero. Research shows that the most appropriate points in the model can be the limits of the measuring range $\left(x_{\min }, x_{\max }\right)$. In this case the parameter $B$ will be determined by the formula

$$
B=\frac{f_{r}\left(x_{\max }\right)-f_{r}\left(x_{\min }\right)}{x_{\max }-x_{\min }} .
$$

The graphical appearance of the solution of the task of the construction of a straight for the static characteristic in accordance with the condition (18) that the average error from nonlinearity is zero, is shown in fig.6 (straight 1 ). The problem is solved for the model (17), defining the function of the actual static characteristic.

On fig.7 via curve 1 are represented, in graphical form, the values of the error from nonlinearity, when the task is solved according to the model (19) and curve 2 - the values of the error in solving the problem through model (7), which corresponds to straight 2 from fig. 6 .

Fig. 7 shows that the differences between the values of the error from nonlinearity in solving the task, using respectively models (19) and (7), is not essential, but model (19) gives a better opportunity to automate the process of construction of the straight, determining the nominal static characteristic.

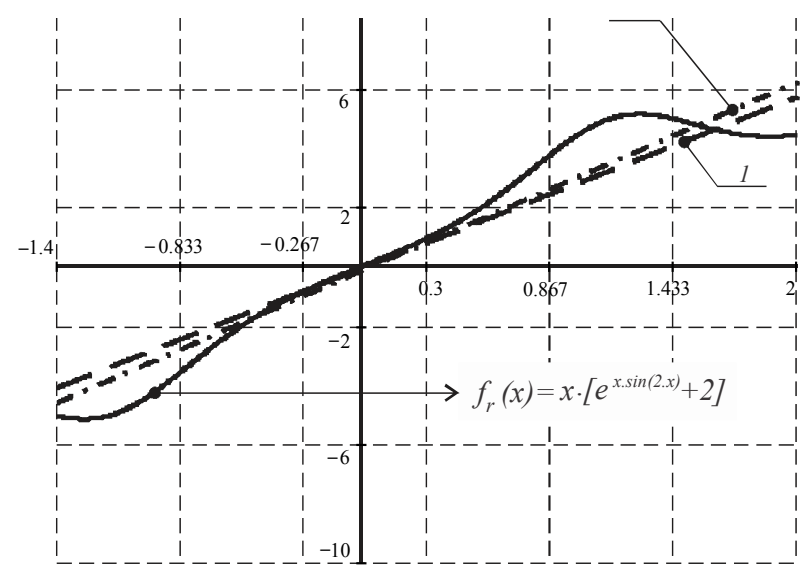

Fig. 6. Constructing the straight according to condition (18)

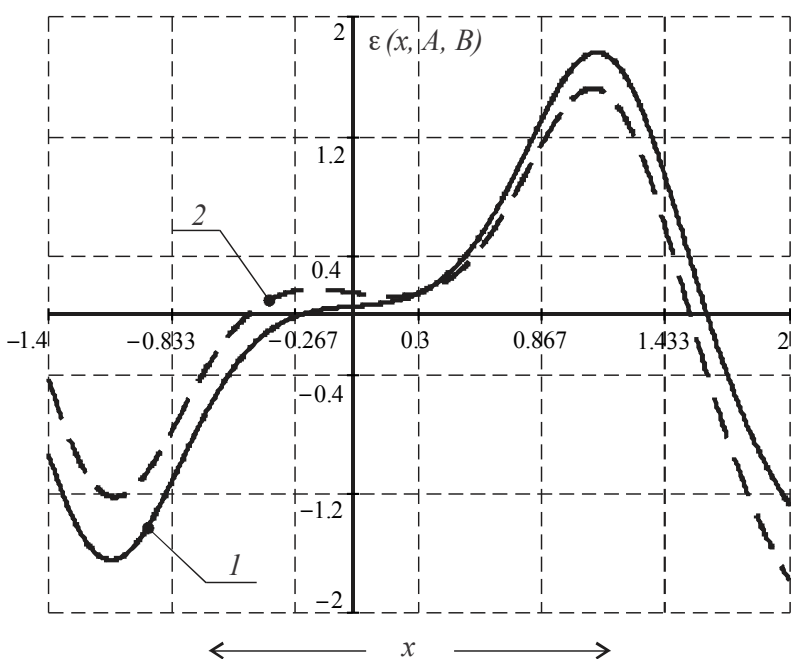

Fig. 7. Values of errors of nonlinearity

\section{Conclusion}

The error from nonlinearity of the static characteristic is one of the most common in the measuring practice constituting of the cumulative error. This error occurs at the end of the metering circuit of the measuring instruments and can be due to instrumental inaccuracies of the parameters, involved in the design scheme of the equipment, as well as due to reasons, conditioned by the imperfection of the measurement method.

In modern measuring devices, the error from nonlinearity of the static characteristic is usually removed in the digital part of the device, where the necessary algorithm for calculation of the measurement results is carried out. Therefore, as a basic condition, is considered less the 
linearity requirements of the static characteristic in the hardware part, as its stability, ie, the immutability of this feature in the changing conditions of actual use. This does not mean however, that the task of assessing the error from nonlinearity of the static characteristic of the measuring instruments has lost its relevance today. As before, the preliminary assessment of this error allows to estimate its value with the permissible error of the measurement results and to adopt informed decisions about the need to use some of the other means of removing the error.
The above presented mathematical models allow the evaluation of the error from nonlinearity in relation to the permissible error of the measurement results and the creation of the necessary algorithms for correction this error. The models were developed, based on the requirements to achieve greater accuracy in the assessing and greater accuracy in the measurements. The structure of the models is in conformity with the conditions for automating the handling procedures in the digital modules of the measuring instruments.

\section{References}

1. Dichev, D., F. Kogia, H. Koev. Models for the Analysis of Static Characteristics of Measuring Instruments. In XXVI National Scientific Symposium: Metrology and Metrology Assurance. Sazopol, Bulgaria, 2016, 57-64.

2. Dichev, D., H. Koev, T. Bakalova, P. Louda. A Model of the Dynamic Error as a Measurement Result of Instruments Defining the Parameters of Moving Objects. Measurement Science Review, Issue 4, Vol. 14, 2014, 183-189, ISSN 1335-8871.

3. Dichev, D., Koev, H., Bakalova, T., Louda, P. A Kalman FilterBased Algorithm for Measuring the Parameters of Moving Objects. Measurement Science Review, 15 (1), 2015, 19-26, ISSN 13358871

4. Jencurakova, D., R. Palencar. The statistical methods for the management of the measurement proces. APLIMAT 2016 - 15th Conference on Applied Mathematics, Bratislava, Slovakia, 2016, 636-643, ISBN 978-802274531-4.

5. Yuling, Z. The Accurate Marketing System Design Based on Data Mining Technology: A New Approach. In AMEII 2015. Zhengzhou, China, 2015, 1952-1956.

6. Dichev, D., H. Koev, T. Bakalova, P. Louda. A Gyro-Free System for Measuring the Parameters of Moving Objects. Measurement Science Review, Issue 5, Vol. 14, 2014, 263-269, ISSN 1335-8871.

7. Dichev, D., H. Koev, T. Bakalova, P. Louda. A Measuring Method for Gyro-Free Determination of the Parameters of Moving Objects. Metrology and Measurement Systems, Issue 1, Vol. 23, 2016, 107 -
118, ISSN 0860-8229.

8. Hegeduš, H., P. Mostarac, R. Malarić. Comparison of RMS Value Measurement Algorithms of Non-coherent Sampled Signals. Measurement Science Review, Issue 3, Vol. 11, 2011, 79-84, ISSN $1335-8871$

9. Jiang, M., J. Luo, D. Jiang, J. Xiong, H. Song, J. Shen. A Cuckoo Search-Support Vector Machine Model for Predicting Dynamic Measurement Errors of Sensors. IEEE Access, Vol. PP, Issue: 99, 2016, 1-7, ISSN 2169-3536.

10. Nilsson, JO, I. Skog. Inertial sensor arrays - A literature review. Navigation Conference (ENC), 2016 European, IEEE, 2016, 1-10, ISBN: 978-1-4799-8915-7.

11. Jermak, C., M. Rucki. Static Characteristics of Air Gauges Applied in the Roundness Assessment. Metrology and Measurement Systems. Issue 1, Vol. 23, 2016, 85-96, ISSN 2300-1941.

12. Hajiyev, C. Sensor Calibration Design Based on D-Optimality Criterion. Metrology and Measurement Systems. Issue 3, Vol. 23, 2016, 413-424, ISSN 2300-1941.

13. Pawlowski, W. Dynamic Model of Oscillation-Assisted Cylindrical Plunge Grinding With Chatter. Journal of Manufacturing Science and Engineering. Issue 5, Vol. 135, 2013, ISSN 1087-1357.

14. Tomczuk, K. A High Pressure Resonator Transducer with a Programmed Correction of Static Characteristics. Metrology and Measurement Systems. Issue 4, Vol. 20, 2013, 667-676, ISSN 2300-1941. 